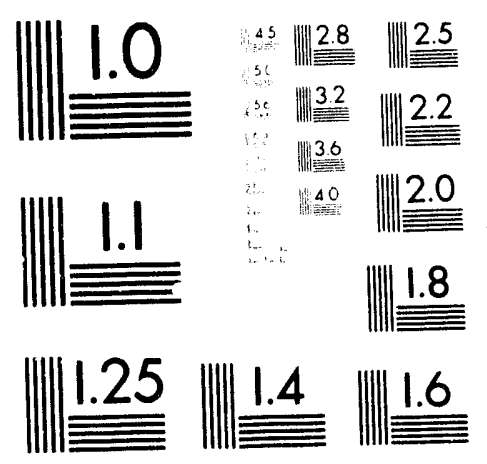



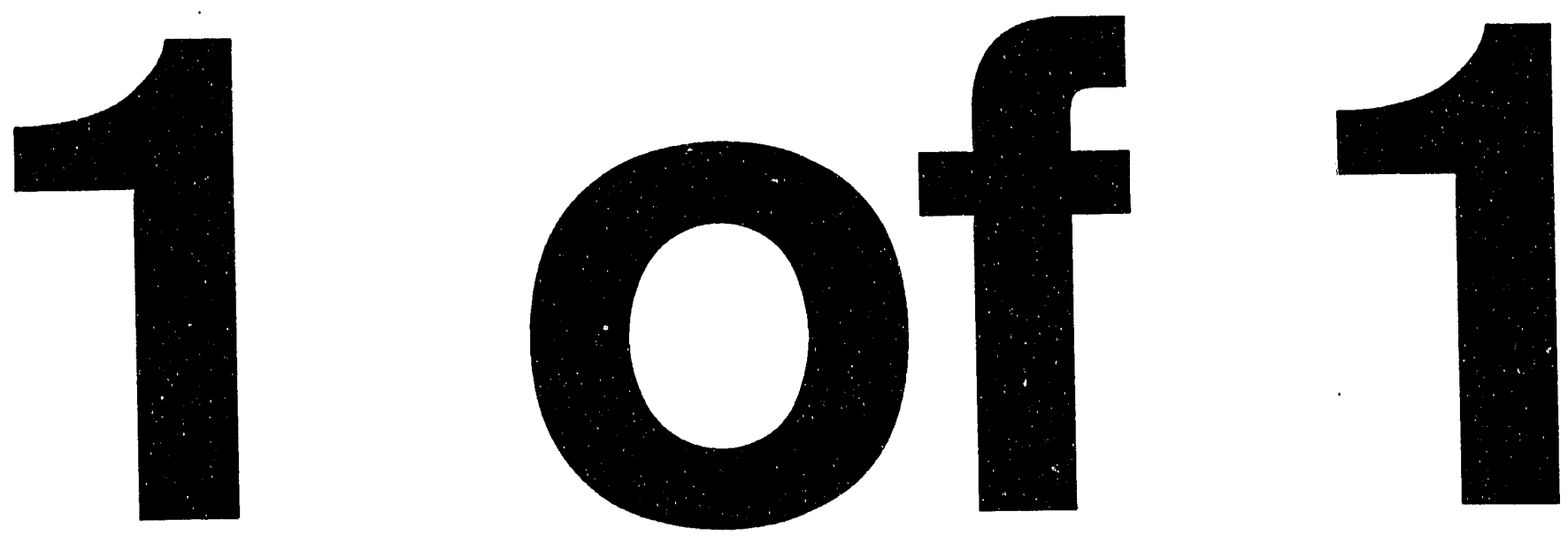
LBL 34575

August 30, 1993

\title{
Restoring Locality with Faster-Than-Light Velocities *
}

\author{
Philippe II. Eberhard \\ Lawrence Berkeley Laboratory \\ University of California \\ Berkeley, CA 94720, USA
}

\begin{abstract}
The idea of "locality" is a deep rooted concept. It does not have to be abandoned even if "loophole free" EPR experiments are performed and confirm the predictions of quantum theory. To satisfy locality, one can imagine that influences at a distance are exerted via mechanisms involving an ether and effects propagating in that ether at a velocity $V>c$. Such model of physical phenomena is not Lorentz invariant but, with $V$ large enough, the model can be made to reproduce the results of all experiments where quantum mechanics and Lorentz invariance have been verified.
\end{abstract}

- This work is supported in part by the Director, Office of Energy Research, Office of High Energy and Nuclear Physics, Division of High Energy Physics of the U.S. Department of Energy under Contract DE-AC03-76SF00098.

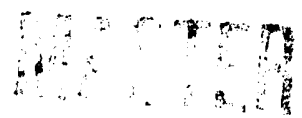




\section{A Historical Precedent}

It is the contention of this paper that loophole free EPR experiments [1] would not rule out all forms of "locality per say," i.e. would not necessarily imply that influences can be exerted at a distance instantaneously without the mediation of an ether. The argument is inspired by a historical precedent, from Newton's times, when the principle of locality was threatened already. In Newton's theory of gravitation, celestial bodies exert attractive forces on each other, instantaneously at a distance and through vacuum, i.e. without an apparent material support to mediate that action at a distance. However, in a letter, Newton expressed himself in the following terms [2] :

...That gravity should be innate, inherent, and essential to matter, so that one body may act upon another at a distance through a vacuum, without the mediation of anything else, by and through which their action and force may be conveyed from on: to another, is to me so great an absurdity that I believe no man who has in philosophical matters a competent faculty of thinking can ever fall into it. ..

That quote shows that, in Newton's times, the existence of "something" to mediate actions at a distance was not an idea that could be disposed of easily, even though the theory of gravity at the time seemed to point in another direction. Today, because predictions of quantum theory concerning the EPR-paradox [3] have been shown to imply instantaneous actions at a distance [4], that locality principle is in jeopardy again, but, now as in Newton's time, it is natural that one look for possibilities to hang on to that principle.

There was a remarkable development of gravitation theory, long after Newton's letter, when that theory was modified and did not involve instantaneous actions at a distance anymore. In "general relativity" indeed, gravitational effects propagate in gravitational waves at the finite velocity $c$. General relativity is in agreement with experimental results of today, but it is also compatible with astronomical observations that justified Newton's gravitation theory, because the latter observations had measurement errors which made the difference between instantaneous and finite-speed actions at a distance unnoticeable.

In this paper, it is suggested that the same circumstances may apply again to the present interpretation of EPR experiments [5] against locality and that this can be true even if and when a loophole free EPR experiment [1] is performed, if and when it demonstrates the existence of actions propagating faster than $c$. Then 
experiments that have confirmed Lorentz invariance and restricted relativity can be reinterpreted in order for the theory to cope with those superluminal velocities. To demonstrate this point, it is sufficient to construct one model that accounts for all experimental data to date and is local. Such a model exists [6]. It is not claimed to yield the correct description of phenomena in nature but it is an example to prove the argument.

\section{The Local Model}

\subsection{Description of Reality}

The model uses operators in Fock space. Let $\psi_{1} \ldots \psi_{n}$ be any set of vectors in Fock's space. The $\psi_{k}$ 's are not necessarily normalized to 1 . The model uses operators in Fock space of the same mathematical form as density matrices of quantum mechanic.s

$$
\begin{gathered}
M=\sum_{k} \psi_{k} \psi_{k}^{\dagger}, \\
\operatorname{Tr}\{M\}=1 .
\end{gathered}
$$

At each point of space of coordinates $x$ and at time $t$, there is an operator called the "quantum-state matrix," $\underline{Q}(x, t)$, of the form of $M$ of Eq. (1) and satisfying Eq. (2). It describes reality at the point of coordinates $x$ at time $t$ and contains all the information that is available there. $Q(x, t)$ is a local quantity. It does not depend on the origin of coordinates of space and time.

As in quantum mechanics, an observable is associated with a set of projection operators. The probability $p_{j}$ of observing outcome \#j of a measurement performed at $x$ and $t$ is given by a trace

$$
p_{j}=\operatorname{Tr}\left\{\underline{\Pi}_{j} \underline{Q}(x, t)\right\},
$$

where the $\underline{\Pi}_{j}$ are projection operators which depend on the kind of measurement performed but not on $x$ and $t$. The projection operator $\underline{\Pi}_{j}$ is related to its counterpart $\tilde{\Pi}_{j}$, in the Heisenberg representation of quantum mechanics, by the relation

$$
\underline{\Pi}_{j}=e^{-i H t} e^{i P x} \tilde{\Pi}_{j} e^{-i P x} e^{i H t},
$$

where $P$ is the total momentum and $H$ the Hamiltonian operators as they are defined in quantum field theory. Equation (4) makes $\underline{\Pi_{j}}$ an operator independent of translations of the origin of space and time coordinates, unlike $\tilde{\Pi}_{j}$. 
Let us define the operator

$$
\tilde{Q}(x, t)=e^{i H t} e^{-i P x} \underline{Q}(x, t) e^{i P x} e^{-i H t} .
$$

$\tilde{Q}(x, t)$ is a mathematical quantity that is useful to compare the predictions of the model with those of quantum mechanics. It cannot be used to describe a local reality at $x$ and $t$ because, unlike $\underline{\underline{C}}(x, t)$, it does depend on the origin of coordinates of space and time.

Eqs. (3) and (4) show that the probability $p_{j}$ of outcome \#j is the same in the model and in quantum mechanics if the density matrix in Heisenberg representation is [7] :

$$
\rho=\tilde{Q}(x, t),
$$

$x$ and $t$ being the space coordinates and the time of the measurement.

\subsection{Evolution of $\underline{Q}(x, t)$}

$Q(x, t)$ evolves in times as the result of two effects, the "Schroedinger evolution" and the "collapse phenomenon." Most of the time, $\underline{Q}(x, t)$ evolves according to the equation

$$
\frac{\partial \underline{Q}(x, t)}{\partial t}=-i(H \underline{Q}(x, t)-\underline{Q}(x, t) H) .
$$

This is the "Schroedinger evolution." Since $P$ and $H$ commute, it follows that, as long as the evolution is of the Schroedinger type between two times $t_{0}$ and $t$, $\tilde{Q}(x, t)$ is constant in time and, if Eq. (6) is satisfied for some $x$ at some initial time $t_{0}$, it is also satisfied at time $t$. Then, for a measurement made at point $x$ at time $t, \mathrm{Eq}$. (3) yields the same predictions as quantum mechanics [7].

From time to time, $\underline{Q}(x, t)$ evolves according to a "collapse" scenario. This happens when a measurement is performed, but it can be made to occur more often if the model is asked to reproduce the results of other models of quantum mechanics where there are spontaneous wave-function collapses without the presence of observers [8]. In any case there is a point $m$ with space coordinates $x_{m}$ where the collapse first occurs at a time $t_{m}$ and there are projection operators $\underline{\Pi}_{m, i}$. If the collapse is initiated by a measurement, $m$ and $t_{m}$ are the point and the time at which the measurement occurs and $\underline{I}_{m, i}$ is the projection operato associated with the measurement outcome. The collapse mechanism first affects 
$\underline{Q}\left(x_{m}, t_{m}\right)$ at point $m$

$$
\underline{Q}\left(x_{m}, t_{m}+\epsilon\right)=\left(\frac{1}{p_{m, i}}\right) \underline{\Pi}_{m, i} \underline{Q}\left(x_{m}, t_{m}-\epsilon\right) \underline{\Pi}_{m, i},
$$

where

$$
p_{m, i}=\operatorname{Tr}\left\{\underline{\Pi}_{m, i} \underline{Q}\left(x_{m}, t_{m}-\epsilon\right)\right\} .
$$

After the time $t_{m}$, there is a propagation of the collapse in space at a velocity $V$, which is a parameter yet of unknown value in the model, but which may be larger than $c$. At each point of space coordinates $x$, there is a time $t_{c}(x),\left(t_{c}(x) \geq t_{m}\right)$, at which a collapse occurs following the measurement at $m$

$$
t_{c}(x)=t_{m}+\frac{\left|x-x_{m}\right|}{V} .
$$

Local (differential) equations can be written for $\underline{Q}(x, t)$ and the propagation of the collapse phenomenon, [6], in such a way that, for all $x$ 's and in extremely good approximation,

$$
\tilde{Q}\left(x, t_{c}(x)+\epsilon\right)=\left(\frac{1}{q_{m, i}}\right) \tilde{\Pi}_{m, i} \tilde{Q}\left(x, t_{c}(x)-\epsilon\right) \tilde{\Pi}_{m, i},
$$

where

$$
q_{m, i}=\operatorname{Tr}\left\{\underline{\Pi}_{m, i} \tilde{Q}\left(x, t_{c}(x)-\epsilon\right)\right\},
$$

which are the same rules as those given for the evolution of density matrices during a measurement [7]. Therefore, if $\widetilde{Q}(x, t)$ satisfies Eq. (6) during the Schroedinger evolution that preceded $t_{c}(x), \tilde{Q}(x, t)$ satisfies it also during the Schroedinger evolution that follows $t_{c}(x)$.

\section{Comparison with Quantum Mechanics}

\subsection{Quasi-Equivalence}

If, in the model, one sets the parameter $V$ at $\infty$, collapses everywhere occur at time $t_{c}(x)=t_{m}$, i.e. at the same time as in quantum mechanics. If, in addition, Eq. (6) is verified at all $x$ 's at an initial time $t_{0}$, Eq. (6) will be satisfied every time later. Then Eq. (3) will yield the same probabilities as quantum mechanics. 
Actually the condition of Eq. (6) for all $x$ 's at the initial time $t_{0}$ can be removed. ${ }^{1}$ In quantum mechanics, all experiments first require a "preparation" of the quantum system under study. This preparation consists of preliminary observations which determine the initial conditions well enough to make the predictions meaningful. In mathematical terms, this means that the density matrix $\rho_{0}$ before the preliminary observations is in principle unknown, but the preparation induces collapses of that density matrix $\rho_{0}$ into another, $\rho$, where the ambiguities caused by our uncertainties about $\rho_{0}$ can be neglected. The matrix $\rho$ is the one involved in the measurments later on. All that is predicted by quantum mechanics are correlations between the observations made during the "preparation" and those made during the "measurements."

In the model with $V=\infty$, consider a preparation at time $t_{0}$ involving a measurement at a point of coordinates $x_{0}$ and a second measurement at time $t$ at a point of coordinates $x$. Assume $\tilde{Q}\left(x, t_{0}-\epsilon\right)$ to be different from $\tilde{Q}\left(x_{0}, t_{0}-\epsilon\right)$. According to the procedure described above, the probability $p_{0, \ell}$ of outcome \# $\ell$ in the "preparation" and the subsequent conditional probability $p_{j}^{(\ell)}$ of outcome $\# j$ in the "measurement" will be

$$
\begin{aligned}
& p_{0, \ell}=\operatorname{Tr}\left\{\tilde{\Pi}_{0, \ell} \tilde{Q}\left(x_{0}, t_{0}-\epsilon\right)\right\}, \\
& p_{j}^{(\ell)}=\left(\frac{1}{q_{0, \ell}}\right) \operatorname{Tr}\left\{\tilde{\Pi}_{j} \tilde{\Pi}_{0, \ell} \tilde{Q}\left(x, t_{0}-\epsilon\right) \tilde{\Pi}_{0, \ell}\right\},
\end{aligned}
$$

where $\tilde{\Pi}_{0, \ell}$ is the projection operator associated with outcome \# $\ell$ during preparation, and

$$
q_{0, \ell}=\operatorname{Tr}\left\{\tilde{\Pi}_{0, \ell} \tilde{Q}\left(x, t_{0}-\epsilon\right)\right\} .
$$

These probabilities are the same as those computed in quantum mechanics, if, before preparation, the unknown density-matrix $\rho_{0}$ was assumed to be

$$
\rho_{0}=\sum_{\ell}\left(\frac{p_{0, \ell}}{q_{0, \ell}}\right) \tilde{\Pi}_{0, \ell} \tilde{Q}\left(x, t_{0}-\epsilon\right) \tilde{\Pi}_{0, \ell} .
$$

Thus the same correlations $p_{j}^{(\ell)}$ between preparation and measurements will be predicted by the model and by quantum theory.

If $V$ is not $\infty$ but measurements are spaced in time by intervals larger than $\frac{1}{V}$ times the distance between the points at which the subsequent measurements

\footnotetext{
${ }^{1}$ This had not been noticed at the time Ref [6] was written.
} 
occur, the delay $\frac{|x-x m|}{V}$ in Eq. (10) becomes irrelevant. Consider two measurements, first one in $x_{m}$ at $t_{m}$, then cue at $x$ at time $t$. The quantum state matrix $\underline{Q}(x, t)$, thus $\tilde{Q}(x, t)$, will have been subjected to the collapse of the type of Eq. (11) initiated by the measurement at $x_{m}$ and $t_{m}$ before the measurement at $x$ and $t$ takes place. Since $\tilde{Q}(x, t)$ is constant between collapses, $\tilde{Q}(x, t)$ will have the same value at time $t$ as if the collapse had occurred at time $t_{m}$ instead of $t_{c}(x)$. Therefore all predictions will be the same as if $V=\infty$, i.e. as in quantum theory.

\subsection{Differences with Quantum Theory}

From what is said above, it follows that the only circumstances where the model leads to different predictions than quantum mechanics are ones where there is one measurement at point $x$ at time $t$ after another at point $x_{m}$ at time $t_{m}$, and they are spaced in time such that

$$
\left|t-t_{m}\right|<\frac{\left|x-x_{m}\right|}{V}
$$

Then the probabilities $p_{j}$ for the second measurement are computed using Eq. (3) with a matrix $Q(x, t)$ that has not been subjected to the collapse generated by the first measurement. In the model, the probability $p_{i, j}$ of observing outcomes \# $i$ at the measurement at point $\left(x_{m}, t_{m}\right)$ and $\# j$ at point $(x, t)$ is

$$
p_{i, j}=\operatorname{Tr}\left\{\tilde{\Pi}_{m, i} \tilde{Q}\left(x_{m}, t_{m}\right)\right\} \times \operatorname{Tr}\left\{\tilde{\Pi}_{m, i} \tilde{Q}(x, t)\right\},
$$

which, in general, will be different than the predictions of quantum mechanics.

These probabilities may be also different from those made by other local models where collapses propagate at a finite velocity $V$. However, in all those local models, Bell's inequalities must be holding when Ineq. (17) holds, while they do not always hold for the predictions of quantum theory. Therefore if $V$ is not too large, there is a possibility to detect a failure of orthodox quantum theory in future experiments and our model could account for certain kinds of these violations. The cases where Ineq. (17) is satisfied have been studied in more detail in Ref. [6].

In any event, $V$ is a free parameter of the model and can be assumed to be as large as necessary to make the difference with $V=\infty$ negligible in the atata of all the experiments where quantum mechanics has been verified. Therefore the model is compatible with any tested prediction of quantum theory and the model cannot be ruled out by any past or future experiment where the predictions of quantum theory are verified. The experiment can only set a lower limit for $V$. 


\section{Lorentz invariance}

In relativistic quantum mechanics, if probabilities $p_{j}$ have been computed using a given space-time restframe, the same results will be obtained using the same computation rules in another restframe. For relativistic quantum theory, there is no fundamental property that distinguishes one restframe from another. In this sense, relativistic quantum mechanics is Lorentz invariant.

For the model referred to in this paper, if $V=\infty$, the computation of the probabilities $p_{j}$ can also be carried out using the same rules in all restframes. This is obvious since, in all restframes, the predictions will be the same as in quantum mechanics. However the model does not only provide a procedure to compute the $p_{j}$ 's, it is also supposed to describe reality at each point of coordinates $x$ at time $t$ by the quantity $\underline{Q}(x, t)$. The collapse of $\underline{Q}(x, t)$ at the same time $t_{m}$ for all $x$ 's is not Lorentz covariant. The description of reality by the model needs a special Lorentz restframe where collapses occur at the same time for all $x$ 's. This restframe can be considered to be the restframe of an ether and, in this sense, the model is not Lorent $z$ invariant. However, since the probabilities of observable quantities are invariant, there is no experiment that can tell what the ether restframe is. As in quantum mechanics, any restframe can be used to make the predictions.

If $V$ is finite but larger than $c$, the model is also not Lorentz invariant and one can define the restframe of the ether as the restframe in which collapses propagate with the same velocity $V$ in all directions. If, in the ether restframe, measurements are spaced in time by an interval so large that Ineq. (17) is never satisfied, the probabilities $p_{j}$ are the same as for $V=\infty$, therefore it is not possible to identify the ether restframe. All restframes can be used equally to compute the predictions.

If $V<\infty$ and condition (17) applies to two measurements, then the predictions for the second measurement are different from what they are for $V=\infty$, therefore from those of quanuum mechanics. These differences exist only if condition (17) is fullfilled in the ether restframe and, for $V>c$, condition (17) is not Lorentz invariant. One can study experimentally the space-time conditions under which the correlations between two observables measured at two different locations are different from those predicted by quantum mechanics, and determine what the ether restframe is. This would be the best way to illustrate the violation of Lorentz invariance in nature if the predictions of the model are correct. However this restframe cannot be identified if condition (17) is never fulfilled in a way that can be seen in the data of an experiment.

In conclusion, the model provides a frame work to test both quantum mechanics 
and Lorentz invariance. In addition, before any violation is found, the model can be used by anyone agreeing with Newton's above quoted statement and wanting to justify his belief in "locality."

\section{References}

[1] N.D. Mermin, "The EPR experiment - thoughts about the 'Loophole,'" New Techniques and and Ideas in Quantum Measurement Theory, edited by D.M. Greenberger (New York Academy of Science, New York, 1986) pp. 422 428.

[2] I. Newton in a letter to R. Bentley, 1692/3, quoted in Theories of the Universe. M.K. Munitz, ed., The Free Press, New York, (1957) 217.

[3] A. Einstein, B. Podolsky and N. Rosen, Phys. Rev. 47 (1935) 777.

[4] J S. Bell, Physics 1 (1964) 195; followed by generalizations : J.F. Clauser, M.A. Horne, A. Shimony and R.A. Holt, Phys. Rev. Lett. 23 (1969) 880; H.P. Stapp, Phys. Rev. D 3 (1971) 1303; J.F. Clauser and M.A. Horne, Phys. Rev. D. 10 (1974) 526; J.S. Bell, CERN preprint Th 2053, reproduced in Epistem. Lett. (Assoc. F. Gonseth CP 1081, CH-205 Bienne) 9 (1975) 11.

[5] S.J. Freedman and J.F. Clauser, Phys. Rev. Lett. 28 (1972) 938; J.F. Clauser, Phys. Rev. Lett. 37 (1976) 1223; E.S. Fry and R.C. Thompson, Phys. Rev. Lett. 37 (1976) 465; A. Aspect, P. Grangier, and G. Roger, Phys. Rev. Lett. 47 (1981) 460; ibid. 49 (1982) 91; A. Aspect, J. Dalibard, G. Roger, Phys. Rev. Lett. 49 (1982) 1804; and many others.

[6] P.H. Eberhard, in Quantum Theory and Pictures of Reality, W. Schommers, ed., Springer Verlag, Heidelberg (1989) 169.

[7] J. Von Neuman, Mathematical Foundations of the Quantum Mechanics Princeton University Press, Princeton (1955).

[8] G.C. Ghirardi, A. Rimini and T. Weber, Phys. Rev. D 34 (1986) 470; G.C. Ghirardi, P. Pearle, and A. Rimini, Phys. Rev. D 42 (1990) 78. 

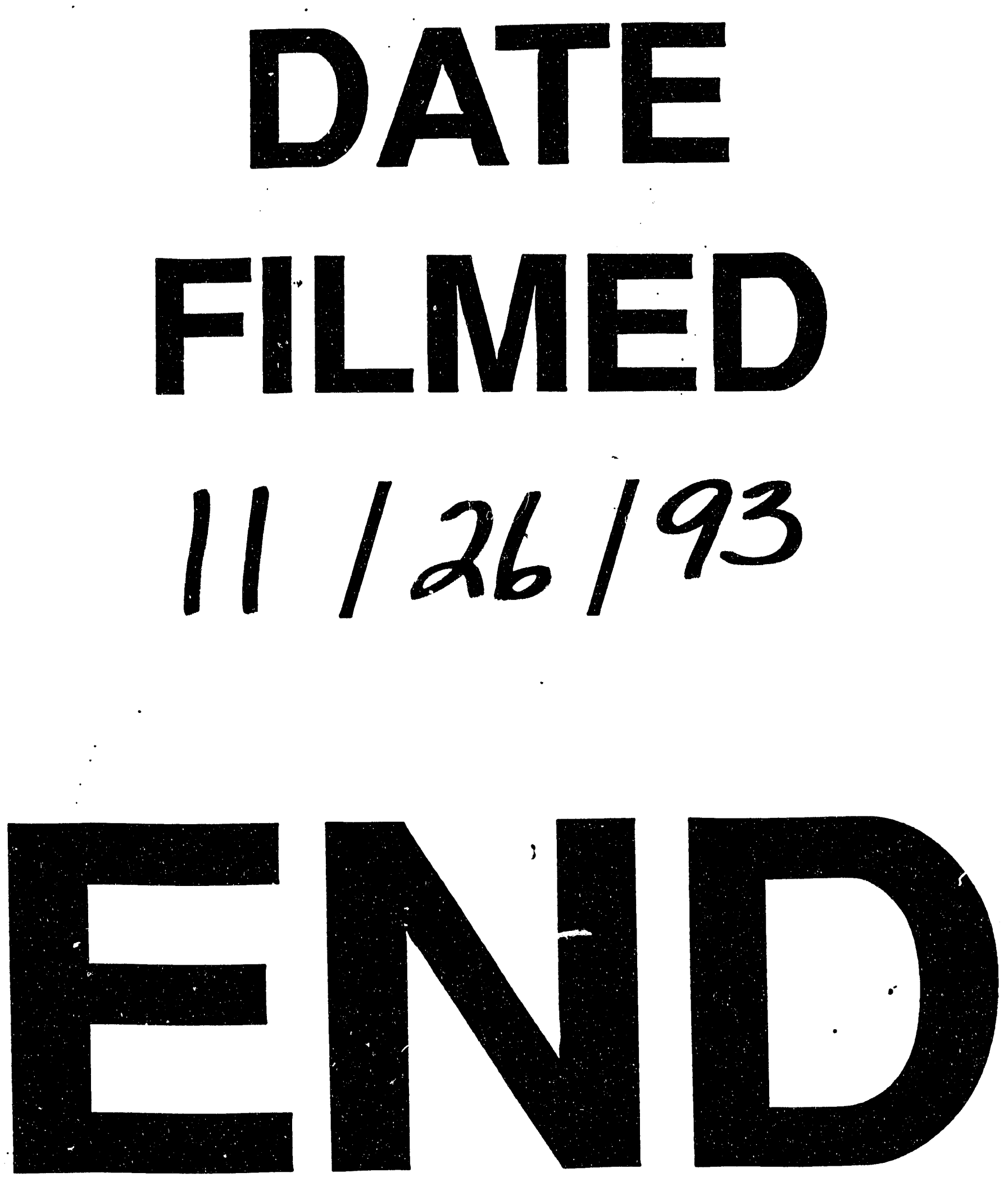
